Classification

Physics Abstracts

$07.80-78.70 \mathrm{D}-78.90$

\title{
EXELFS as a structural tool for studies of low $Z$-elements
}

\author{
Virginie Serin, Gerald Zanchi and Jean Sévely \\ CEMES-LOE, BP 4347, 31055 Toulouse Cedex, France
}

(received May 22, 1992; accepted June 22, 1992)

\begin{abstract}
Résumé. - Des études de pertes d'énergie d'électrons effectuées en microscopie électronique à moyenne et haute tension nous ont permis de mettre en évidence les modulations EXELFS sur les distributions associées aux excitations du niveau atomique $\mathrm{K}$ des éléments de faibles numéros atomiques $(Z \leq 16)$. Ces modulations constituent une source d'information importante sur l'ordre atomique local dans les matériaux légers. Après un rappel des bases physiques et des principes de traitement du signal EXELFS, nous discutons les aspects essentiels de la technique expérimentale dans son application en microscopie électronique à transmission à moyenne tension. Les possibilités de la technique sont étudiées à travers trois types de résultats obtenus à partir d'échantillons de graphite, de nitrure de bore hexagonal, de carbone amorphe et de carbure de silicium cubique. Ces exemples ont été choisis de manière à discuter la précision de la technique, les possibilités de détermination de l'ordre à courte distance dans la matière cristalline et amorphe et à faire apparaître, en particulier, l'influence des effets de diffusion multiple dans les échantillons.
\end{abstract}

\begin{abstract}
By using high and medium voltage microscopes we have been able to obtain EELS spectra showing EXELFS modulations above the $\mathrm{K}$ edge of low atomic number $Z \leq 16$, elements. These modulations supply important structural information about the local atomic environment of the absorbing atoms. After recapitulating the physical foundations of EXELFS and the ways of analysing the spectra, we discuss the main steps of the experimental technique, as developed for the medium voltage transmission electron microscope. The potential of the technique is studied for four types of specimen : graphite, hexagonal boron nitride, carbon made amorphous and cubic silicon carbide. They have been chosen to illustrate the accuracy of the method, its potential for the determination of short-range order of crystallized and non-crystallized materials and to emphasize the influence of plural scattering effects on the RDF determination.
\end{abstract}

\section{Introduction.}

During the last years, Electron Energy Loss Spectroscopy (EELS) experiments have demonstrated that spectra from thin films can be obtained with enough signal to make EXtended Energy Loss Fine Structure (EXELFS) treatment possible [1 - 4]. The EXELFS signal appears as an oscillatory structure superimposed on the high-energy-loss side of an ionization edge in electron energy loss spectra. Such modulations can extend from a few tens eV up to several hundreds eV 
past the edge and may have a magnitude of $10 \%$ of the signal [5 - 7].

This development of EXELFS may be attributed to the importance of the microstructural information it makes accessible and to recent improvements in the techniques associated with electron microscopy.

It has also been pointed out that the counting rates achievable with electron scattering can be competitive with those obtained from synchrotron radiation experiments, provided the core edge lies below about $3 \mathrm{keV}$. Furthermore, for the lower atomic number elements, with the core edge lying below $1 \mathrm{keV}(Z \leq 11)$, EXELFS becomes more efficient than EXAFS. The electron technique has a further advantage in that it can be carried out on a microscopic region by choosing a fine electron probe on the sample $(10 \mathrm{~nm})[8,9]$.

The purpose of this paper is to recall the main aspects of the technique from the theoretical and experimental points of view and to discuss its potential in terms of some applications in the domain of low $Z$ element compounds.

\section{Physical foundation of EXELFS.}

The number of incident electrons of energy $E_{0}$ which are scattered after inelastic scattering associated with a core-level electron excitation is directly dependent on the angular scattering crosssection per unit solid collection angle and per unit path. It is directly given by the Fermi gold rule as proposed by Bethe $[10,11]$.

$$
\frac{\mathrm{d} \sigma_{o n}}{\mathrm{~d} \Omega}=\frac{2 \pi}{\hbar v}\left|<\mathbf{k}_{n}, n\right| H_{\mathrm{e}-\mathrm{a}}\left|\mathbf{k}_{o}, o>\right|^{2} \frac{\mathrm{d} \mathcal{D}}{\mathrm{d} \Omega}
$$

where $\mathbf{k}_{o}$ and $\mathbf{k}_{n}$ represent the incident electron wave vectors, $o$ and $n$ denote the atom state before and after the collision respectively, $\frac{d \mathcal{D}}{\mathrm{d} \Omega}$ is the number of electron states in the continuum per unit energy and solid angle $\Omega, v$ the velocity of the incident electron and $H_{\mathbf{e}-\mathbf{a}}$ is the hamiltonian for the incident electron-atom interaction

$$
H_{\mathbf{e}-\mathbf{a}}=\frac{e^{2}}{\mathbf{R}-\mathbf{r}_{\mathbf{a}}}=\frac{e^{2}}{\mathbf{r}}
$$

where $\mathbf{R}$ and $\mathbf{r}_{\mathrm{a}}$ are the position vectors for the incident and the core electron, respectively.

The cross-section can be written :

$$
\mathrm{d} \sigma_{o n}=\frac{2 \pi e^{4}}{m c^{2}} \frac{1}{\beta^{2}} \frac{\hbar k_{n}}{\hbar k_{o}}\left|\eta_{o n}(\mathbf{k})\right|^{2} \frac{\mathrm{d} Q}{Q^{2}}
$$

where $Q$ is the change of the ejected electron kinetic energy in the collision and

$$
\eta_{o n}(\mathbf{k})=\left\langle n\left|\mathbf{i k} \cdot \mathbf{r}_{\mathbf{a}}\right| o>\right.
$$

In the case of the dipole approximation,

$$
\left|\eta_{o n}(\mathbf{k})\right|^{2}=k^{2}|<n| i \varepsilon_{k}, \mathbf{r}_{\mathbf{a}}|o>|^{2}
$$

where $\varepsilon_{k}$ is the unit vector in the $\mathbf{k}$ wave vector direction. For EELS experiments as developed in the TEM, this expression shows that for small $\mathbf{k}$ values, where the dipole approximation is valid, the electron scattering cross-section has a clear resemblance to the photoabsorption cross-section which is proportional to $[12,13]$

$$
|<n| \mathbf{r} . \varepsilon|o>|^{2}
$$


where $\varepsilon$ represents the electric field vector.

The form of the interaction matrix elements in the two expressions of the cross-section shows the analogy between X-ray absorption and electron energy loss extended fine structures [1]. It is experimentally confirmed by the similarity between the two types of spectra, as shown by comparing EXAFS and EXELFS spectra on the aluminium K-edge and silicon K-edge, already published in the literature, for instance Lafon et al [14] and Zanchi et al [15] for silicon carbide, and by Fontaine $e t$ al [16] and Leapman $e t$ al [1] in the case of aluminium. An identical number of modulations above about $50 \mathrm{eV}$ after the inner shell edge appears at the same frequencies. They are in the two cases related to variations in the matrix elements which are produced by modifications in the final state wave function $|n\rangle$, caused by neighbouring atoms. They are attributed to the interference between the outgoing excited inner-shell electron waves and the electron waves backscattered by the neighbouring atoms [13]. The mathematical treatment already developed for EXAFS can be applied to EXELFS in the same way.

It is considered that the part of the spectrum beyond the K-edge can be written as the product of the single atom spectrum and a factor $(1+\chi(E))$ where $\chi(E)$ corresponds to the EXELFS structure and represents modulations of the spectrum as a function of the energy loss $E . \chi(E)$ is related to $\chi(k)$ using the relationship between $E$ and the wave number $k$ of the ejected electron :

$$
k^{2}=\frac{2 m\left(E-E_{\mathrm{s}}\right)}{\hbar^{2}}
$$

where $E_{\mathrm{s}}$ is the threshold value of the ionization edge. The interference amplitude $\chi(k)$ in $k$ space is given by the formula:

$$
\chi(k)=-\sum_{j} \frac{N_{j}}{k R_{j}^{2}} \mathrm{e}^{-2 \sigma_{j}^{2} k^{2}} \mathrm{e}^{-2 R_{j} / \lambda(k)}\left|F_{j}(k)\right| \sin \left(2 k R_{j}+\varphi_{j}(k)\right),
$$

where $N_{j}$ is the number of the atoms surrounding the central atom in the $j$ th coordination shell at a distance $R_{j}$.

$F_{j}(k)$ is the backscattering amplitude from each of the $N_{j}$ neighbouring atoms of the $j$ th shell.

$\sigma_{j}$ is the Debye-Waller factor which takes into account the thermal vibration and statistic disorder of the atoms $j$.

$\lambda(k)$ is the range of the ejected electron. It is a function of the kinetic energy,

$\varphi_{j}(k)$ is the total phase shift experienced by the ejected electron $[17,18]$. It is given by

$\varphi_{j}(k)=\varphi_{\mathrm{a}}(k)+\varphi_{\mathrm{b}}(k)-\pi$, where $\varphi_{\mathrm{a}}(k)$ is the phase shift due to the central atom and $\varphi_{\mathrm{b}}(k)$ is the phase shift due to the backscattering on the neighbouring atom $j$.

\section{EXELFS signal analysis.}

The EELS data are typically analysed and the Radial Distribution Function (RDF) extracted by following the original EXAFS procedure for the analysis of the as-observed EXELFS signal $[1-7]$. There are two approaches :

- a Fourier transform method allows the RDF to be determined;

- as successfully shown by the EXAFS analysis, a curve-fitting method of a calculated function to the experimental data allows more accurate informations on the RDF to be extracted.

These procedures involve several steps which are extensively discussed in the literature $[1,19,20]$.

The EXELFS spectra are typically recorded in an energy window covering about $400 \mathrm{eV}$ above the edge with an energy resolution better than $3 \mathrm{eV}$ which is sufficient for the accuracy of the results. The treatment of the signal leads to the RDF. The procedure is as follows : 
1) Background subtraction: the smooth background of the spectrum which lies below the inner shell excitation signal is removed from the experimental spectrum, assuming it follows a power law in energy $\left(A E^{-r}\right)$ before and after the edge.

2) Extraction of the oscillatory component : $\chi(E)$ is isolated from the resultant signal by subtracting it from a model of the single atom spectrum. This model consists of a monotonically decreasing $3^{\text {rd }} \rightarrow 5^{\text {rd }}$ order polynomial function.

3) Scale conversion : $\chi(E)$ data are converted to $\chi(k)$ using the equation $k^{2}=\frac{2 m e}{\hbar^{2}}\left(E-E_{\mathrm{s}}\right)$, which connects the ejected electron wave vector $k$ with the incident electron energy loss $E$.

4) RDF determination: The RDF is obtained by Fourier transforming the windowed $k^{n} \chi(k)$ signal where $n=1,2$ or 3 . Each peak in $\operatorname{RDF}(r)$ corresponds to an atomic shell around the central atom. The contribution of each shell to the signal can be isolated through a Fourier filtering procedure. This step is important for the backtransformation of the $\operatorname{RDF}(r)$ and more accurate determination of each mean shell radii.

Special attention must be paid to three main points in the data processing.

- The first point concerns the determination of the energy threshold $E_{\mathrm{s}}$ of the edge because of its strong influence on the zero position and on the calibration of the $k$-scale and thus on the peak positions of the $\operatorname{RDF}(r)$. The choice of this energy zero for the ejected electron is dependent of many parameters and particularly on the chemical state of the atom. As an example, increasing $E_{\mathrm{s}}$ by $3 \mathrm{eV}$ increases the position of a peak near $1.5 \AA$ by $0.01 \AA$ and a peak near $4 \AA$ by $0.02 \AA$.

A solution proposed by some authors [17] consists in adjusting $E_{\mathrm{s}}$ until the main peak in the $\operatorname{RDF}(r)$ coincides with a peak in the imaginary part of the Fourier transform of $\chi(k)$.

- The second point concerns the problem of the windowing of the $k \chi(k)$ signal in order to exclude the near-edge structures related to band structures and plasmon convolution effects. Typically, the windowing smoothly removes modulations about $25 \mathrm{eV}$ above the edge and extends over a range of 8 or $9 \AA^{-1}$. The width of the window function has a strong effect upon the width of the peak in the radial distribution function and thus on the effective resolution of the method.

- The last point concerns the phase shift $\varphi(k)$ experienced by the ejected electron.

As suggested by Lee and Beni [17], a phase shift can be represented by a function $\varphi(k)=$ $a+b k+c k^{2}+d / k$. The values of $a, b, c$ and $d$ can be deduced from tabulated values of $\varphi(k)$ for the atoms considered [18].

The major term in this equation is the term linear in $k$. This term is responsible of the shift of the peak positions in the $\operatorname{RDF}(r)$. This linear part of the phase shift is estimated from the data of Teo and Lee [18] for the central and backscattering atom and added to the peak positions of the $\operatorname{RDF}(r)$.

These steps lead to a determination of $R_{j}$ values with an accuracy which can be of $\pm 0.02 \AA$.

\section{EXELFS experimental techniques.}

Due to the relatively low value of the probability of exciting the core electrons, corresponding to one thousandth of the inelastic scattering cross-section, the detection of the EXELFS modulations requires optimized experimental conditions. This consideration determines the main characteristics of the experimental aspects of the EXELFS technique.

The detection of the EXELFS signal is a statistical problem and requires a signal-to-noise ratio better than one percent when recording the associated core-edge. This is achieved with a counting rate higher than 10000 counts per channel in that part of the spectrum [1]. The basic experimental parameters which allow us to maximize the signal are : the intensity of the incident electron beam, the collection angle of the spectrometer and the detection system. Recent improvements 
in microscope design and performance have made the technique more efficient for that purpose [21].

- Concerning the electron beam intensity, the $\mathrm{LaB}_{6}$ filament is now commonly used in the microscope, and the beam current intensity typically available is then higher than $30 \mu \mathrm{A}$. This intensity allows us to extract sufficient elemental information from nanometre scale areas of the specimen. A small electron probe, a few nanometres in diameter, can be focused at the level of the object plane. It defines the selected area for the analysis when the spectrometer is used in the diffraction mode (image coupling). This procedure is preferred to working in the image mode where, due to strong chromatic aberration effects of the image forming system, the accuracy of the area selection via the spectrometer entrance aperture is limited. This is of particular importance when using a high magnification for the image and when recording core excitation edges in the 500 - $3000 \mathrm{eV}$ energy loss range [22].

- The whole of the information in the electron beam transmitted by the object can be collected by choosing a sufficiently wide spectrometer entrance aperture. This aperture defines the collection semi-angle of the spectrometer. It can be chosen large enough to collect all the information. Its typical value may well be far larger than the characteristic scattering angle $\theta_{E}=E / \gamma m_{0} . v^{2}$ which ensures a sufficiently high collection efficiency. The large convergence angle of the incident probe makes the angular and energy resolution of the spectrometer worse. This is a less critical parameter for EXELFS analysis performance where a $3 \mathrm{eV}$ resolution preserves acceptable accuracies for the interatomic distance determination.

- An important step in the potential use of the technique has been achieved with the development of the new types of linear parallel detectors employed in the Gatan PEEL spectrometer. This recording mode allows all the electrons which emerge from the spectrometer to be collected and preserves a high detective quantum efficiency (D.Q.E.), better than 0.5, for the detection [23].

Other parameters of importance are related to the specimen thickness and the incident energy which are correlated if one considers the effect of plural scattering contributions on the spectrum profile.

Because of plural scattering the jump ratio of an edge and the edge visibility fall rapidly as the specimen thickness increases. On the other hand, plural scattering may produce extra peaks above the threshold, which may cause inaccuracies in the EXELFS analysis [1]. These effects can be reduced either by using thin enough specimens or by application of a Fourier transform deconvolution procedure to the spectra. It has been shown that the EXELFS signal is not strongly reduced as long as the $t / \lambda_{\mathrm{p}}$, the ratio of the thickness $t$ to the inelastic mean free path $\lambda_{\mathrm{p}}$, is lower than 0.3 [21].

By using the lowest possible reduced thickness it is possible to optimize the EXELFS signal itself, and thereby to extract the more accurate information. This is achieved either by using thin samples or by increasing the incident energy. As an example, $\lambda_{\mathrm{p}}$ is increased by a factor about 1.8 at $300 \mathrm{keV}$ relative to $100 \mathrm{keV}$ incident electrons [24].

The discussion of these different parameters shows that the use of medium voltage microscopes, equipped with a parallel detection energy loss spectrometer, provides favourable conditions for the development of the EXELFS technique.

For instance, with the CM30 ST Philips microscope, which is currently in use for our present experiments, the main parameters are chosen in order to meet as far as possible these conditions. The EXELFS structures which can be studied are observed on the K-edges of elements ranging from boron $(Z=3)$ to sulphur $(Z=16)$. The incident energy is $300 \mathrm{keV}$. The condition $t / \lambda_{\mathbf{p}} \leq$ 0.3 then corresponds to a thickness of the sample below about $40 \mathrm{~nm}$ for samples containing these light elements. The probe size of the electron beam on the specimen chosen for an adequate counting rate, is about $100 \mathrm{~nm}$. It determines the spatial resolution of the analysis.

Choosing a $2 \mathrm{~mm}$ spectrometer aperture, a recording time below 1 minute is sufficient to detect 
the K-edges with a counting statistics in the order of $50000 \mathrm{cts} / \mathrm{channel}$. This depends on the collection angle $\alpha$, which is changed by varying the microscope camera length $L$. For the $2 \mathrm{~mm}$ aperture $\alpha$ varies between 4 and 9 mrad when changing the camera length $L$ between 450 and 900 $\mathrm{mm}$. These conditions correspond to the recording of the carbon and silicon K-edges, respectively.

\section{Results.}

The results illustrate problems of three kinds. First, through the example of graphite and hexagonal boron nitride, which have well-established structures, the potential of the technique is discussed. In this case very low $\mathrm{K}$ shell energies $(285 \mathrm{eV}$ and $188 \mathrm{eV}$ for carbon and boron respectively) are involved. They have not been investigated by EXAFS and they are typical of the energy loss range accessible by EELS. The second set of results gives an example of short-range order determination in the case of amorphous carbon obtained by crack deposition. The results can be compared to the case of graphite. In the third type of experiment we point out the effect of plural scattering associated with the thickness on the EXELFS treatment results.

The well known structure analogy between graphite and hexagonal BN and the implications of these types of elements in recent aeronautical and space technology lead us to evaluate the structural properties of these materials at the nanometre scale. The EXELFS technique has been used to characterize these structures. The EXELFS signal associated with the carbon and boron $\mathrm{K}$ edge is shown before and after processing in the figures 1 and 2 . It provides the first-neighbour distances which are compared to the theoretical distances in tables of figures 1 and 2. Connections have been established between the position and the amplitude of the main maxima of the RDF and the interatomic distances in these two structures. They are interpreted by taking into account the differences between the resolution of the treatments applied to the different EXELFS signals, which are limited to $400 \mathrm{eV}$ for graphite and $200 \mathrm{eV}$ for boron in BN, because of the occurrence of the nitrogen $\mathrm{K}$ shell excitation at $400 \mathrm{eV}$. This implies an accuracy of $0.04 \AA$ in the RDF of graphite and $0.06 \AA$ in the RDF of boron in BN.

The graphite structure is well identified, with three distances in the same 002 plane, while there is a major spacing at $3.60 \AA$ between the planes. In the case of $\mathrm{BN}$, due to the poorer resolution associated with a lower $k$ window, only three main distances are observed. The first two maxima are associated with distances in the hexagonal plane, while the $3.46 \AA$ value corresponds to an interplane spacing. The gap, which is observed between graphite and boron nitride on the distances at $3.46 \AA$ in hexagonal $B N$ and at $3.63 \AA$ in graphite, is understood by considering the different stacking of the respective hexagonal planes, as shown figures $3 \mathrm{a}$ and $3 \mathrm{~b}$. This difference is characterized by variations in the value of $N_{j} / R_{j}^{2}$ associated with the fourth and fifth coordination shells of carbon and boron in the respective materials.

In addition, the relative intensity of the peaks of the RDF reflects the combined effect of different parameters : finite aperture size, variation of $k$ as a function of energy and orientation. The inversion of the intensity of the peaks corresponding to in-plane and interplane distances about 1 $\AA$ and $3 \AA$ respectively, which is observed in figures $1 \mathrm{~b}$ and $2 \mathrm{~b}$, can be interpreted by taking this last effect into account. In the case of $\mathrm{BN}$ in figure $2 \mathrm{a}$, the strong peak at $190 \mathrm{eV}$ is attributed to $1 \mathrm{~s} \longrightarrow \pi^{*}$ band transitions, while the peak at $200 \mathrm{eV}$ corresponds to $1 \mathrm{~s} \rightarrow \sigma^{*}$ band transitions $[25,26]$. Such a near-edge fine structure is indicative of the orientation of the $c$ axis in the BN crystal, parallel to the momentum transfer vector $\hbar q$, where $q$ is given by $q=\mathbf{k}_{n}-\mathbf{k}_{o}[27,28,26]$. Within this orientation, the RDF is expected to have a major peak at about $3 \AA$, which unites the $3.33 \AA$ and $3.63 \AA$ interplane distances in $B N$. On the other hand the carbon Kedge signal in graphite in figure 1a exhibits a small $\pi^{*}$ peak which is indicative of a non parallel orientation of the momentum transfer vector $k$ to the $c$ axis. This may explain the intensity of the first distance 

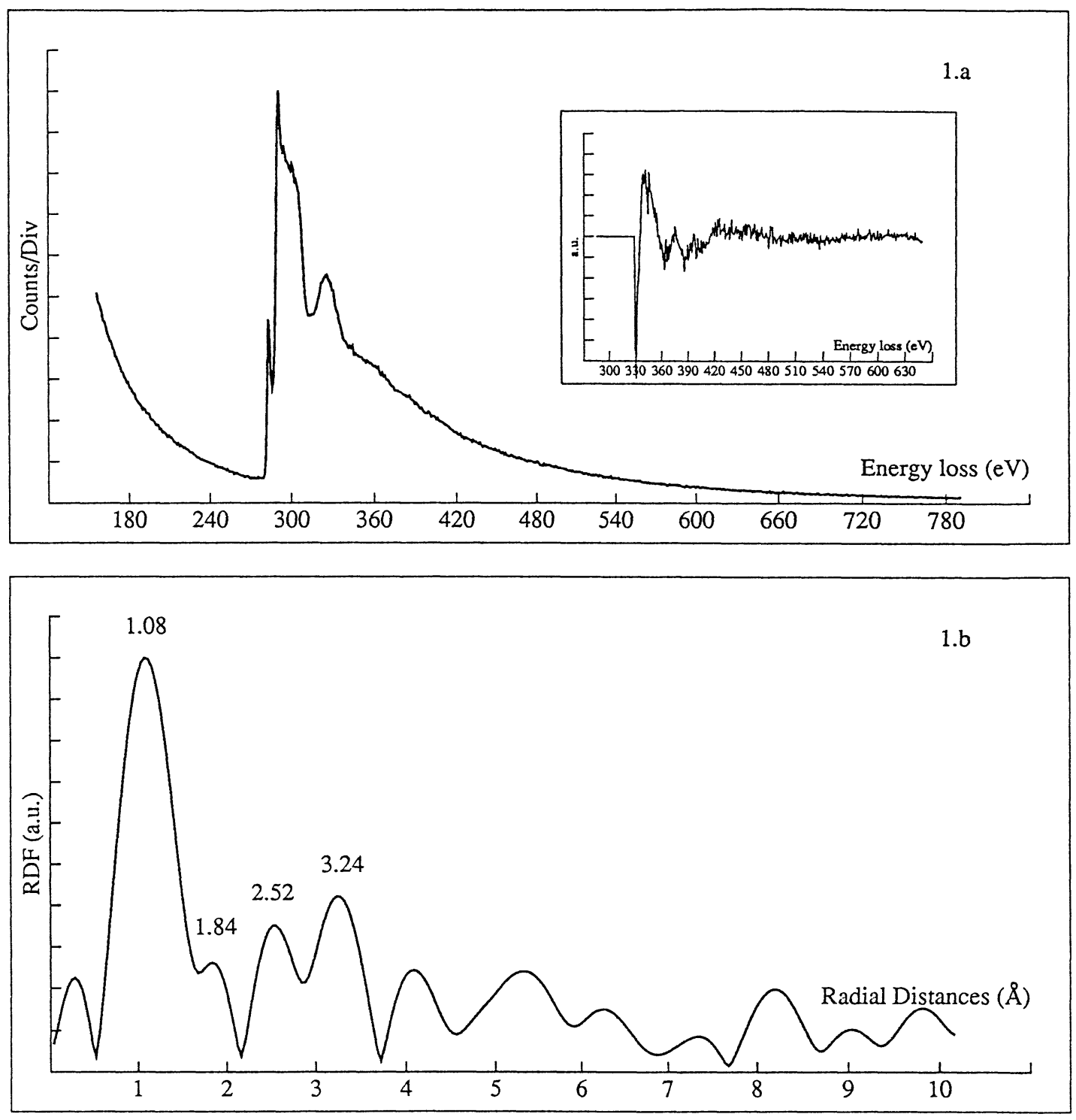

\begin{tabular}{|c|c|c|c|c|c|c|c|}
\hline $\begin{array}{l}\text { Theoretical } \\
\text { Graphite }\end{array}$ & $\begin{array}{l}R_{j}(\AA) \\
N_{j} \\
N_{j} / R_{j}^{2} \\
\end{array}$ & $\begin{array}{c}1.42 \\
3 \\
1.49\end{array}$ & $\begin{array}{c}2.45 \\
6 \\
0.99\end{array}$ & $\begin{array}{c}2.85 \\
3 \\
0.37\end{array}$ & $\begin{array}{c}3.35 \\
0 \text { ou } 2 \\
0.09\end{array}$ & $\begin{array}{c}3.63 \\
12 \text { ou } 6 \\
0.68\end{array}$ & $\begin{array}{c}3.76 \\
6 \\
0.42\end{array}$ \\
\hline $\begin{array}{l}\text { Experimental } \\
\text { Pyrographite }\end{array}$ & $R_{j}(\AA)^{*}$ & 1.44 & 2.20 & 2.88 & & 3.60 & \\
\hline
\end{tabular}

* corrected for the $0.36 \AA$ phase shift value

Fig. 1. - a) Fine structures observed on the carbon $\mathrm{K}$ edge at $300 \mathrm{kV}$ with a pyrographite sample and from an area about $10 \mathrm{~nm}$ in diameter. The inset shows the modulations $\chi(E)$ extracted from this spectrum. b) Magnitude of the associated RDF. The table allows us to compare the $R_{j}$ radial distances deduced from the EXELFS treatment, with the known $R_{j}$ radial distances in graphite. $N_{j}$ represents the associated coordination numbers. 

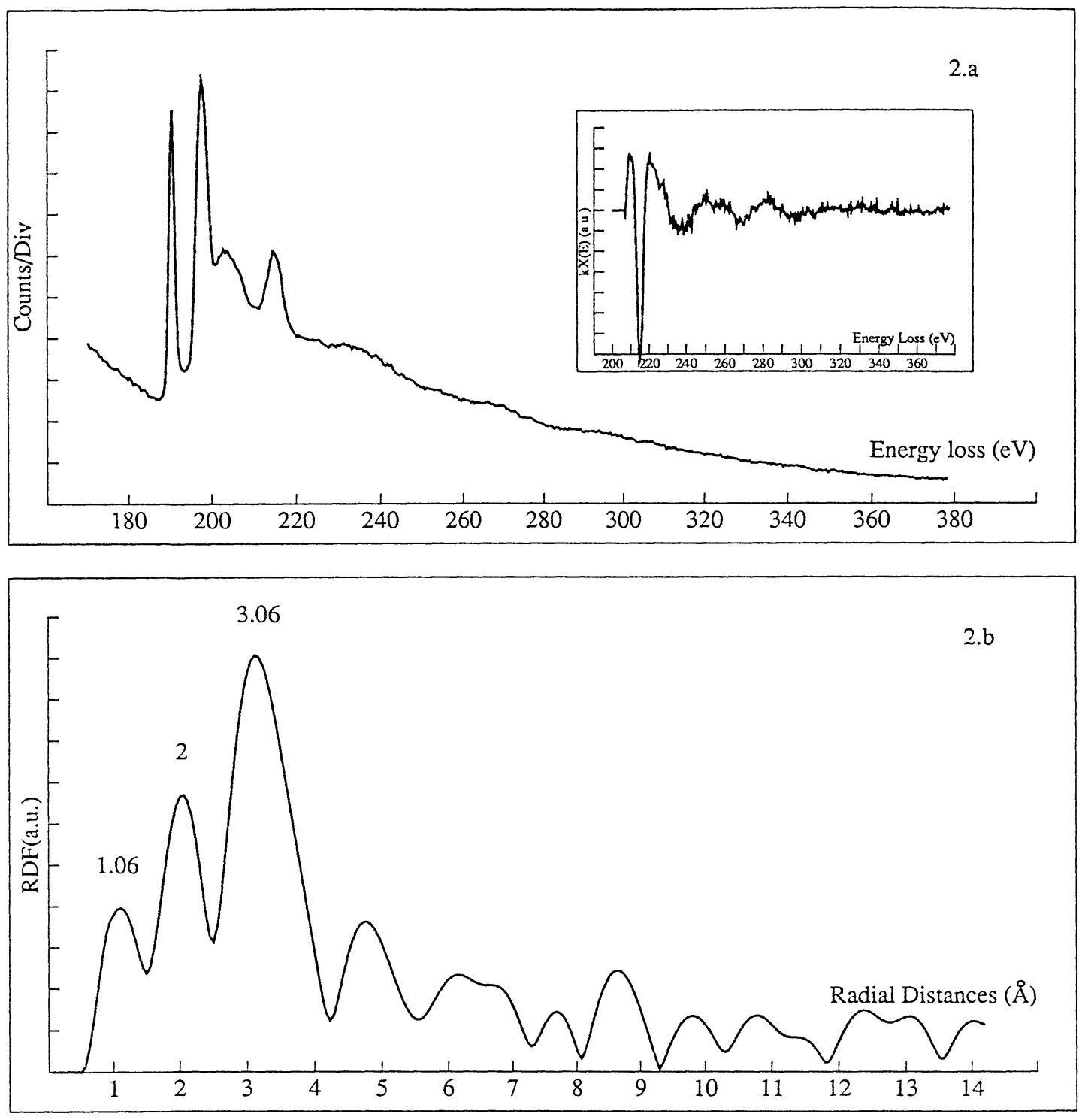

\begin{tabular}{cl|c|c|c|c|c|c}
\hline Theoretical & $R_{j}(\AA)$ & 1.44 & 2.50 & 2.89 & 3.33 & 3.63 & 3.81 \\
$\mathrm{BN}$ & $N_{j}$ & 3 & 6 & 3 & 2 & 6 & 6 \\
& $N_{j} / R_{j}^{2}$ & 1.44 & 0.96 & 0.36 & 0.18 & 0.45 & 0.41 \\
\hline $\begin{array}{c}\text { Experimental } \\
\mathrm{BN}\end{array}$ & $R_{j}(\AA)^{*}$ & 1.42 & 2.43 & \multicolumn{6}{|c}{3.46} \\
\hline
\end{tabular}

* corrected for the phase shift value $(0.36 \AA$ for $\mathrm{B}-\mathrm{N}$ and $0.43 \AA$ for $\mathrm{B}-\mathrm{B})$

Fig. 2. - a) Energy loss spectrum of hexagonal BN around the boron $K$ edge. The inset shows the modulations $\chi(E)$ extracted from this spectrum. b) Boron RDF obtained from the spectrum. The table allows us to compare $R_{j}$, the radial distances around the boron atoms deduced from the EXELFS treatment, with the known $R_{j}$ distances in hexagonal $\mathrm{BN}$. 
in the RDF of graphite, which is determined in these measurements. It is in agreement with the results given by Disko et al [28].

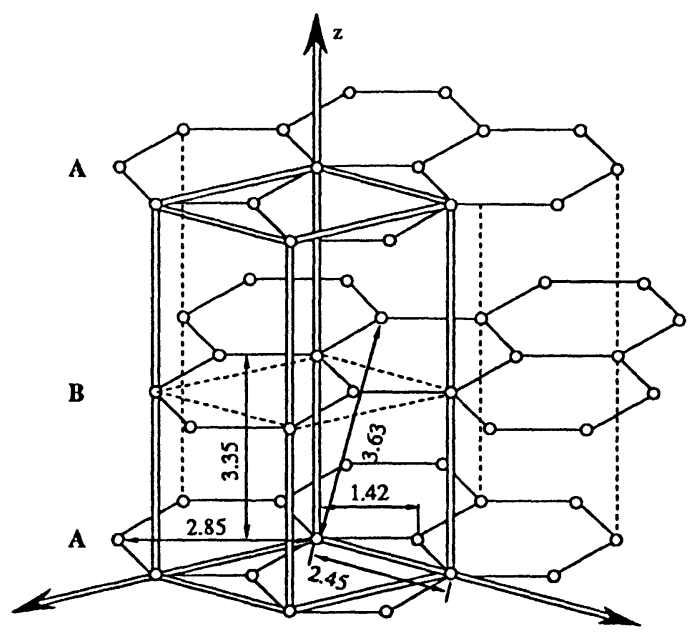

a)

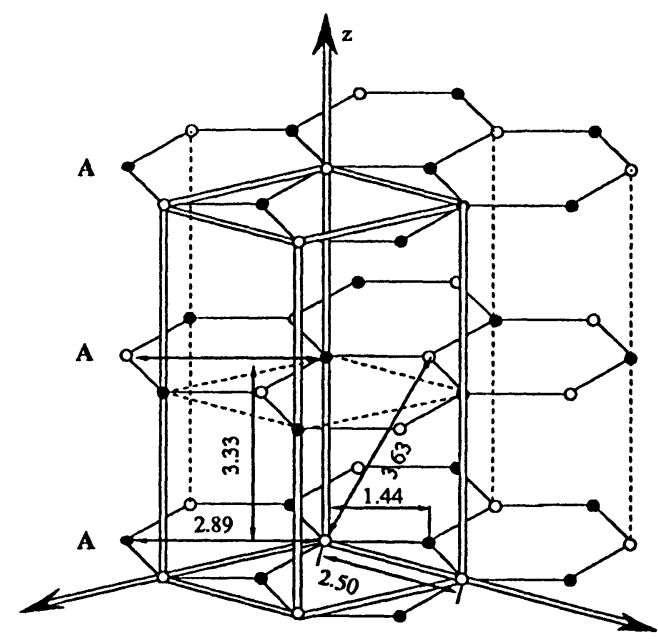

b)

Fig. 3. - a) Structure of graphite with the first interatomic distances around C (o) atoms. b) Hexagonal BN with the first interatomic distances around B (०) atoms, $(\bullet)$ correspond to nitrogen atoms.

In the case of the carbon specimen, made amorphous by crack deposition, the carbon $\mathrm{K}$ edge and the EXELFS structure which can be extracted are shown figure 4. From the treatment, figure 4b, two main distances at $1.34 \AA$ and $2.49 \AA$ are deduced. Their values corrected for the $0.36 \AA$ experimental phase shift value are : $1.70 \AA$ and $2.85 \AA$ [29]. These values are large in comparison with the two first distances in graphite and diamond. They confirm that in carbon made amorphous, the classical crystalline structures are destroyed and they show a short-range order in this type of materials. Comparison with the results already published [29] on the different structures of carbon graphite and diamond shows that an $\mathrm{sp}_{2}$ type hybridization is more likely to be present in this amorphous material ; this conclusion confirms some results obtained by Raman spectrometry concerning carbon amorphization [30].

The results on silicon carbide correspond to $\mathrm{SiC}$ specimens, which were commercialy available as single crystals. They were prepared for transmission electron microscopy studies in the classical way, by ion milling, and the specimen shows variable thickness. The results of the as-recorded silicon $\mathrm{K}$ edge is presented in figure 5a and the calculated RDF, which can be directly deduced, is shown figure $5 \mathrm{~b}$. The two first peaks at $1.48 \AA$ and $2.64 \AA$ respectively are attributed to the Si-C and $\mathrm{Si}$-Si interatomic bonds, at $1.86 \AA$ and $3.08 \AA$ respectively. They allow us to deduce phase shift correction values of $0.38 \AA$ and $0.44 \AA$ for Si-C and Si-Si bonds [31].

From the shape of the original silicon $\mathrm{K}$ edge it is clear that the thickness of the specimen introduces multiple scattering effects in the edge structure. These affect the edge structure by introducing additional oscillations due to the plasmon component. In order to correct the treatment for this effect, a Fourier deconvolution technique has been applied to the spectrum $[32,33]$. The results of the treatment are given in figure $6 \mathrm{a}$ and associated $\operatorname{RDF}(r)$ in figure $6 \mathrm{~b}$. The first two 

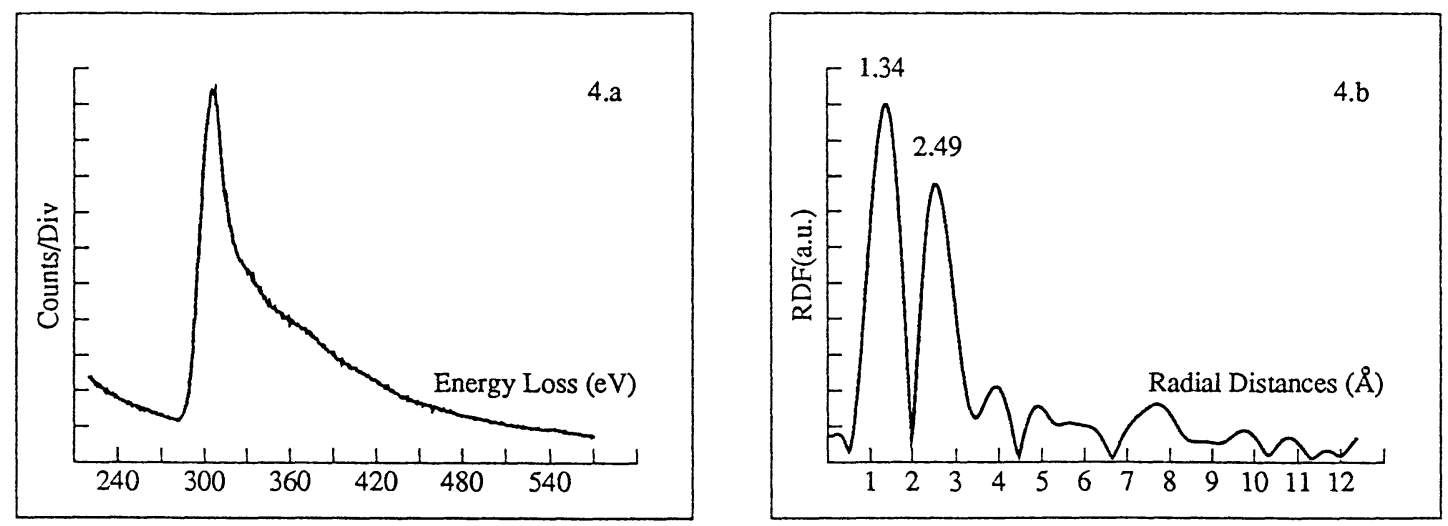

Fig. 4. - a) Plot of the electron energy loss of the carbon K edge corresponding to a thin carbon sample made amorphous by crack deposition. b) Carbon RDF obtained from this spectrum.
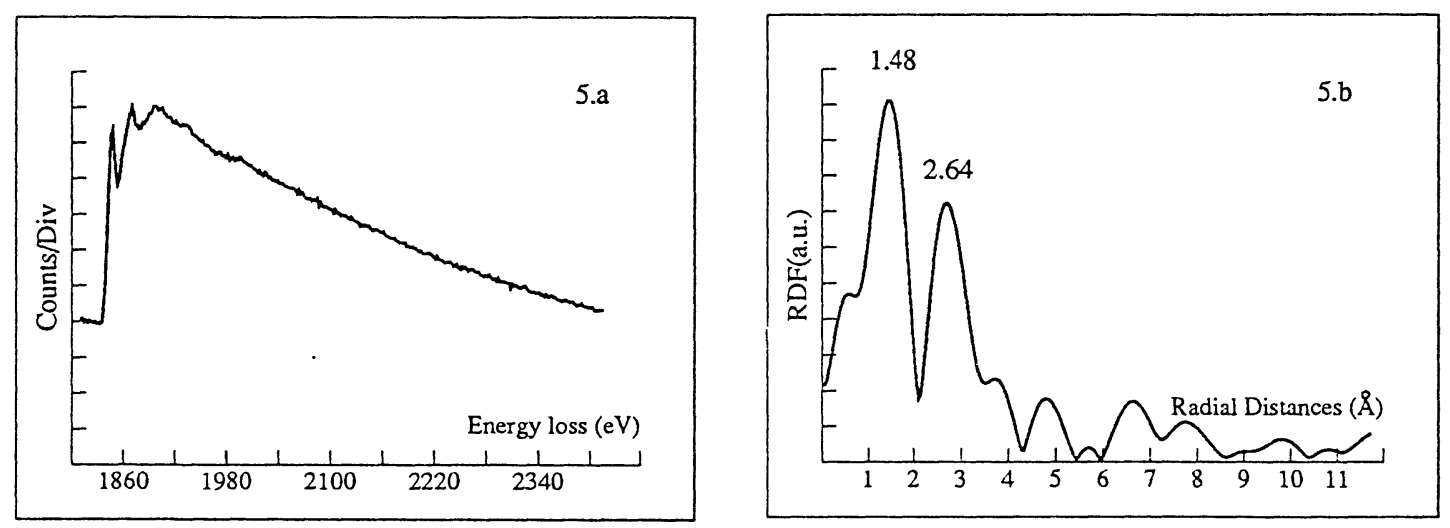

Fig. 5. - a) As recorded energy loss spectrum of the Si K edge obtained with a crystalline $\operatorname{SiC}(\beta)$ sample. $t / \lambda \simeq 0.9$; incident energy : $300 \mathrm{keV}$. b) Silicon RDF obtained from the non-deconvoluted spectrum (5a).

peaks in these data occur in close agreement with values obtained from the non-deconvoluted edge. Their relative intensity is inverted, in better agreement with the proportionality with the factor $N_{j} / R_{j}^{2}$, as can be expected from the general expression $\chi(k)$ for EXELFS. Furthermore, an important maximum is observed at $6.27 \AA$. It should be noticed that a similar distribution has been observed in the $\operatorname{RDF}(r)$ determined by $\operatorname{EXAFS}$ in the $\operatorname{SiC}(\beta)$, where the peak at about $6 \AA$ is attributed to a focusing effect, which corresponds to an enhancement of the amplitude of the peak associated with the fourth shell, around the silicon atoms [14] .

\section{Discussion : future developments and limitations.}

Our EXELFS experiments performed on the recently acquired CM30 ST Philips electron microscope equipped with a Gatan PEELS spectrometer confirm the main conclusion already proposed by authors involved in the exploitation of EXELFS techniques for the studies of materials. The 

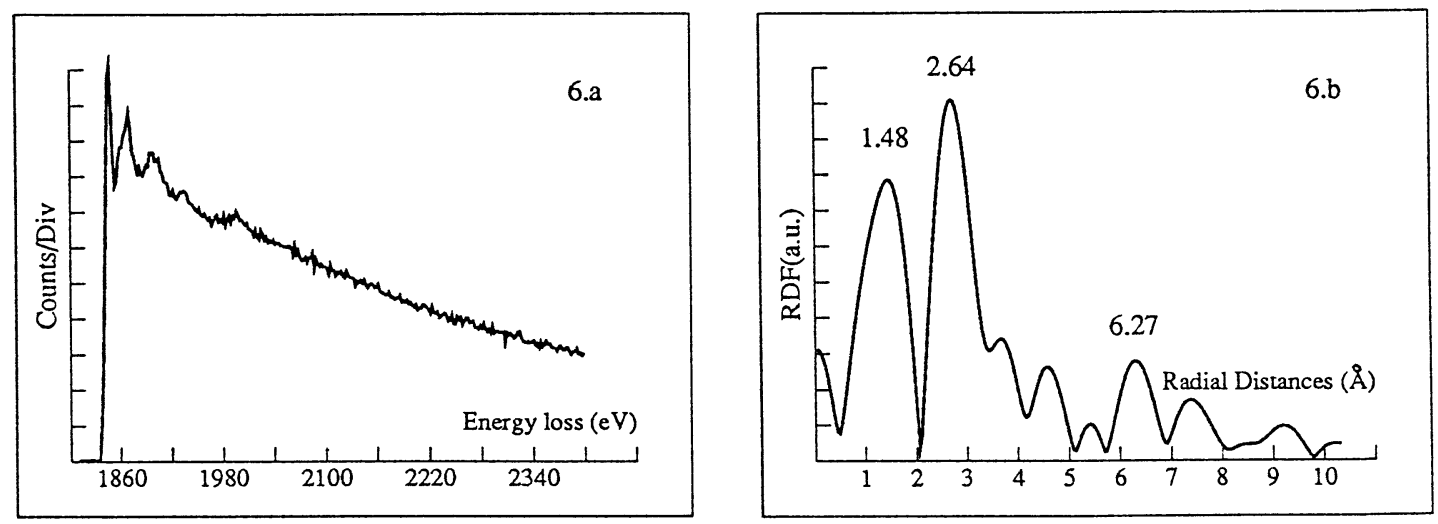

Fig. 6. - a) Deconvoluted energy loss spectrum of the Si K edge shown in the figure 5. b) Silicon RDF obtained from the deconvoluted spectrum (6a).

interest of EXELFS is that it complements EXAFS in the determination of short range order with an accuracy of $0.04 \AA$ in the atomic bonding of crystalline and amorphous materials.

The main advantages are as follows : i) EXELFS is sensitive to low $Z$ elements, and in particular $Z \leq 16$ (sulphur), considering the treatment of the Kedge. ii) The investigation of the sample can be performed with high spatial resolution so that inhomogeneous materials can be studied. This kind of information is combined with that provided by the other methods available on the electron microscope, which include electron diffraction and high resolution imaging. iii) An orientation dependence of the amplitude in the RDF can be predicted from the expression for the excitation cross-section. It may be of interest for a more precise understanding of materials with anisotropic layer structure.

The main limitations of this technique come from the overlapping of inner shell structures in the low $Z$ elements. The example of $\mathrm{BN}$ has shown that overlapping edges may limit the resolution of the treatment to a value of $0.06 \AA$. i) It is clear, that due to the low value of the inner shell excitation cross-section and to the importance of statistical problems for the detection of the EXELFS signal, rather long counting times are necessary. This can be an important limitation for application of the technique for sensitive specimens. ii) Another point concerns the effect of multiple scattering processes on the inner shell edge visibility since they reduce the usable thickness of the sample. Nethertheless, the improvement of the deconvolution technique allow us to extend the range of the sample thickness within which the technique can be applied. It appears that the present improvements in transmission electron microscopy technology, concerning the development of the medium voltage microscope and parallel detection, are favourable for the future use of EXELFS technique for structural studies of materials.

\section{Acknowledgements.}

The authors are indebted to C. Colliex for helpful comments on the manuscript and they thank R. Fourmeaux for its assistance in the experiments.

Ces expériences d'EXELFS s'inscrivent dans le cadre des travaux de spectrométrie de pertes d'énergie d'électrons, développés au laboratoire depuis plus de 20 ans. Ils se sont largement inspirés des idées du Professeur Castaing; nous sommes très heureux de lui exprimer ici notre profonde reconnaissance. 


\section{References}

[1] LeAPMAN R.D., GRUNES L.A., FeJes P.L. and SilCoX J., in EXAFS spectroscopy techniques and applications, B.K. Teo and B.C. Joy Eds (Plenum Press N.Y. 1981) pp. 217-239.

[2] BouRdillon A.J., EL MARSHI S.M., ForTy A.J., Philos. Mag. 49 (1984) 341-352.

[3] TAFRESHi M.A., CSIllag S., YuAN Z.W., BOHM C., Microsc. Microanal., Microstruct., 2 (1991) 515-530.

[4] SERIN V., HSSEIN K., ZANCHI G., SEVELY J., Microbeam Analysis (1990) pp 35-38.

[5] LEAPMAN R.D., COSSLETT V.E., J. Phys.D.9 (1976) L.29-32

[6] COlliex C., CosSLETT V.E., LEAPMAN R.D., TREBBia P., Ultramicroscopy 1 (1976) 301-315.

[7] BATSON P.E., CRAVEN A.J., Phys. Rev. Lett. 42 (1979) 893-897.

[8] ISAACSON M., UTLAUT M., Optik 50 (1978) 213-234.

[9] Kincaid B.M., MeIXner A.E., Platzman P.M., Phys. Rev. Lett. 40 (1978) 1296-1299.

[10] BETHE H., Handbuch der Physik, XXIV/I (1933) 491-523.

[11] INOKuTI M., Rev. Mod. Phys. 43 (1971) 297-347.

[12] Fano U., COOPER J.W., Rev. Mod. Phys. 40 (1968) 441-507.

[13] STERn E.A., Phys. Rev. B10 (1974) 3027-3037.

[14] LAFFON C., Thesis Université Paris Sud (1990).

[15] ZANCHI G. , HSSEIN K., SEVELY J., EMAG 87, Analytical electron microscopy (G.N. Lorimer Ed. 1988) pp. 123-125.

[16] Fontaine A., Lagarde P., Raoux D., Estevas J.M., J. Phys. F. Metal Phys. 9 (1979) 2143-2153.

[17] LEE P.A., BENI G., Phys. Rev. B15 (1977) 2862-2883.

[18] TeO B., LeE P.A., J. Am. Chem. Soc. 101 (1979) 2815-2832.

[19] EGERTON R.F., Electron Energy-loss spectroscopy in the electron microscope (New York, Plenum Press, 1986) pp. 278-287.

[20] JOHNSON D.F., CSILLAG S., STERN E.A., Scanning electron microscopy I (1981) 105-115.

[21] SeVEly J., ZANCHI G., KIHN Y., HsSEIN K., Scanning electron microscopy (J. Kirschner, K. Murata, J.A. Venables Eds., sup. I 1987) 179-189.

[22] EGERTON R.F., Electron Energy-loss spectroscopy in the electron microscope (New York, Plenum Press, 1986) pp. 68-79.

[23] KRIVANEK O.L., AHN C.C., KEENEY R.B., Ultramicroscopy 22 (1987) 103-115.

[24] Jouffrey B., SeVely J., Revue Phys. Appl. 11 (1976) 101-111.

[25] LeapMan R. D.,SilcoX J., Phys. Rev. Lett. 42 (1979) 1361-1364.

[26] Martin J.M., Belin M., Mansot J.L., HsSein K., ZanChi G., Sevely J., EUREM 88, Inst. Phys. Conf. Ser., nø93, vol 2 (1988) 175-176.

[27] STEPHENS A.P., BROWN L.M., Quantitative Microanalysis with high spatial resolution, UMIST/Manchester (1981) 152-158.

[28] DiSKO M.M., KRIVANEK O.L., REZ P., Phys. Rev. B 25 (1982) 4252-4255.

[29] SERIN V., Thesis Toulouse (1989).

[30] CARLES R., Private communication (1992).

[31] HsSEIN K., Thesis Toulouse (1988).

[32] EgerTon R.F., CROZIER P.A., Scanning Microsc. Suppl. 2 (1988) 245-254.

[33] LEAPMAN R.P., SWYT C.R., Analytical electron microscopy (R.H. Geiss Ed. San Francisco Press 1981) pp. 164-172. 